\title{
Vom Umgang mit Parasiten
}

Cäcilia Brendieck-Worm, Alexandra Nadig, Yvonne Thoonsen

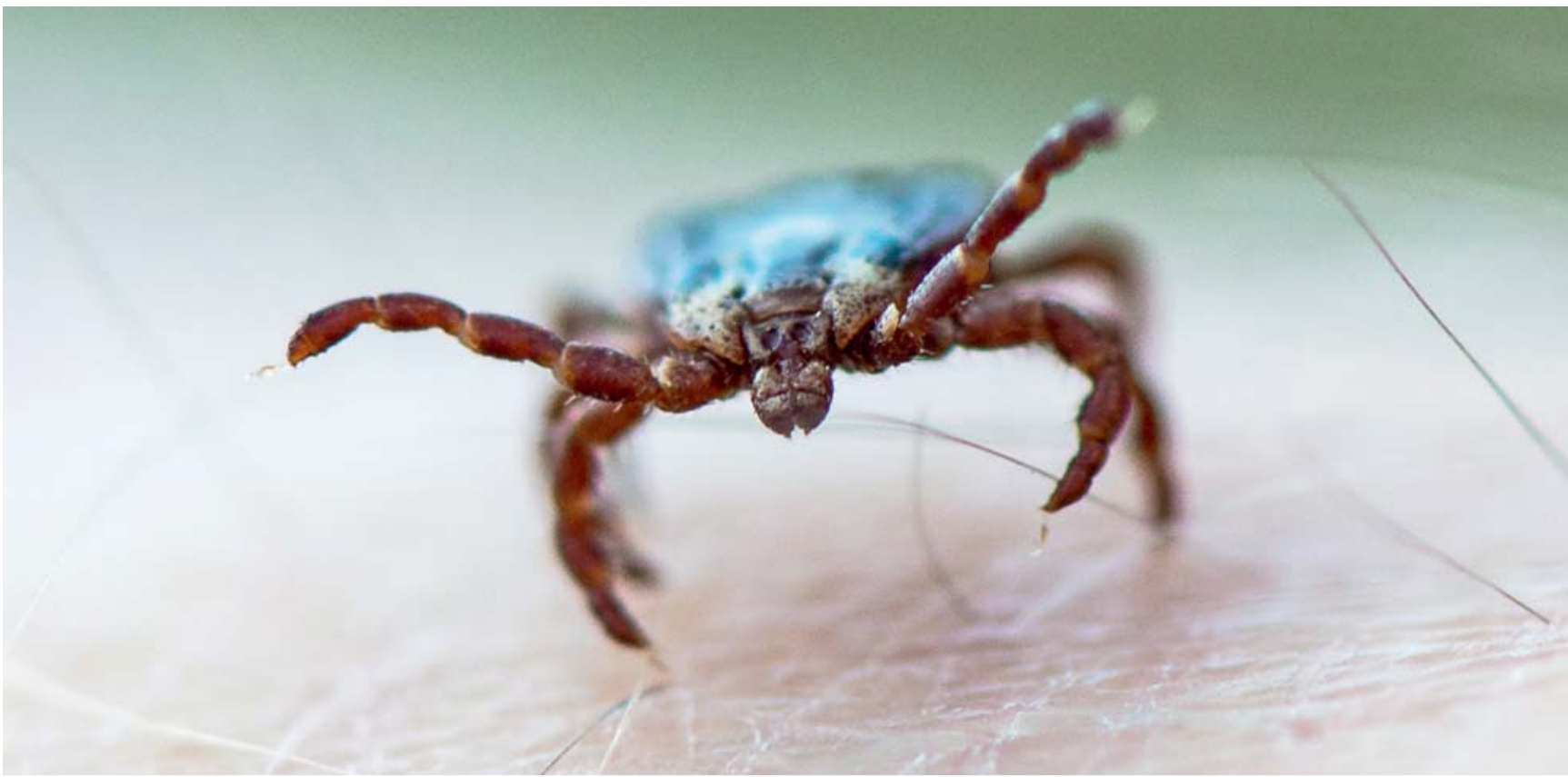

Dermacentor reticulatus, die Auwaldzecke, breitet sich seit 50 Jahren in Deutschland aus, insbesondere im für sie klimatisch günstigen Oberrheingraben. Sie wurde vermutlich durch Hunde aus Ungarn, Österreich und Norditalien eingeschleppt. @ Ferdinand Worm

\section{Parasiten waren Thema im Praxisseminar „Phytos für alle Felle“ des Arbeitskreises Phytotherapie der GGTM in Wettelbrunn 2017. Welche Auswirkungen konventio- nelle Parasitenbekämpfung haben kann - und das nicht nur auf die Haut - ergänzt um Angaben zu Phytotherapeutika werden hier wiedergegeben.}

„Die Flöhe und die Wanzen gehören auch zum Ganzen ...“ (Sprichwort)

Beim konventionellen Umgang mit Parasiten, zu denen wir im Folgenden auch potenziell schädliche Mikroorganismen zählen werden, wird oft außer Acht gelassen, dass praktisch jeder Makroorganismus gemeinsam mit diesen Kleinstlebewesen ein Ökosystem bildet und überhaupt nur innerhalb dieser Gemeinschaft lebensfähig ist. Unter optimalen Bedingungen besteht ein flexibles Gleichgewicht zwischen Parasiten und Makroorganismen, welches das Lebensrecht aller Beteiligten des Systems wahrt. Störungen in diesem Gleichgewicht können für den Makroorganismus gesundheitsgefährdend und sogar lebensbedrohlich werden, denn der Grat zwischen Symbiose, Kommensalismus und schädigendem Parasitismus ist oft schmal. Die Ursachen für derartige Störun- gen sind vielfältig, Lebens- und Haltungsbedingungen und medizinische Maßnahmen spielen dabei zentrale Rollen.

\section{Die Bekämpfung von Ektoparasiten - irrationale Ängste und konkrete Gefahren}

Vom Menschen von alters her besonders gefürchtet sind Zecken, Mücken, Flöhe, Läuse und Wanzen, die als Blutsauger potenzielle Krankheitsüberträger sind (s.S.97 oben). Das macht ihre Bekämpfung besonders wichtig. Doch nicht überall und zu jeder Zeit fungiert ein Parasit auch als Vektor. Sinnvoll ist Bekämpfung nur auf der Basis umfassenden Wissens über die biologischen Zusammenhänge. Es braucht strategisches Vorgehen mit Augen- 
maß, will man nicht durch unüberlegte Bekämpfungsmaßnahmen weitere Schäden anrichten.

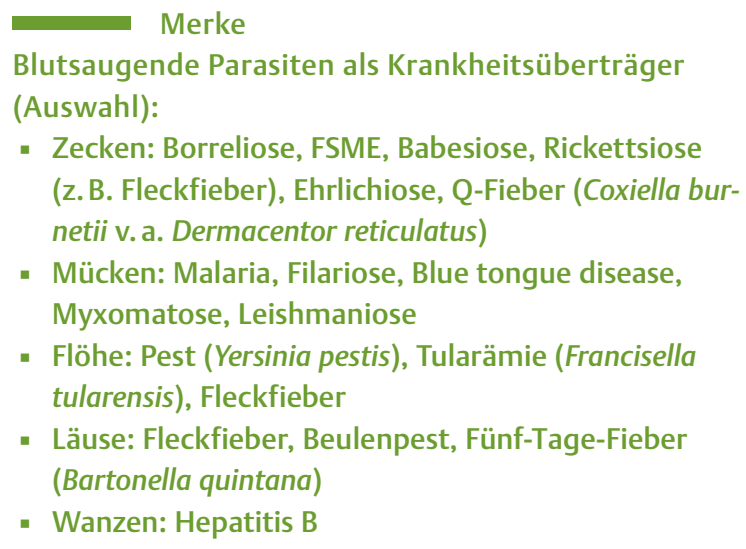

\section{Konventionelle Ektoparasitenbekämpfung}

Gegen Ektoparasiten werden sowohl am Tier (lokal und systemisch) als auch im Umfeld zu den Pestiziden (lat. pestis = Geißel, Seuche und lat. caedere = töten) zählende Ektoparasitika eingesetzt (Akarizide, Insektizide). Ziel ist es, lästige oder schädliche Lebewesen zu töten, zu vertreiben oder in Keimung, Wachstum oder Vermehrung zu hemmen. Eingesetzt werden u.a. neurotoxisch wirkende, zu den Neonicotinoiden zählende Chlornicotinoide, Pyrethroide, zu den makrozyklischen Lactonen gehörende Avermectine und Milbemycine, Phenylpyrazolone wie Fibronyl und Pyriprol sowie organische Phosphorsäureester, die über die Blockade lebenswichtiger Enzyme wirken [20].

\section{Toxizität}

Alle eingesetzten Wirkstoffe sind für Warmblüter mehr oder weniger toxisch (s. Kasten S.98). Insbesondere für Jungtiere unter drei Monaten, kranke, schwache, alte und hochträchtige Tiere besteht die Gefahr der resorptiven Vergiftung. Für Katzen sind etliche Wirkstoffe aufgrund der Glucuronidierungsschwäche lebensbedrohlich toxisch. Es besteht z.T. hohe Ökotoxizität. Fische und nützliche Insekten, insbesondere Bienen werden durch Ektoparasitika gefährdet.

Seit dem spektakulären Tod von 11.000 Bienenvölkern im Oberrheingraben 2008 durch Stäube von mit Neonicotinoiden gebeiztem Getreide, ist die Bienentoxizität dieses Pestizids hinlänglich bekannt. Über die Auswirkungen subletaler Dosen von Neonicotinoiden wird seither kontrovers diskutiert. Was viele Tierhalter nicht wissen: aus dieser Stoffgruppe stammen etliche Produkte zur FlohBekämpfung.

2017 sorgte die Entdeckung des Ektoparasitikums Fipronil in Eiern für Aufregung. Als Spot on oder Spray wird es selbstverständlich für Hund und Katze weiter eingesetzt. Doch selbst bei Anwendung von Kombinationsprodukten aus mehreren chemische Ektoparasitika, lässt sich Zeckenbefall nicht immer verhindern.

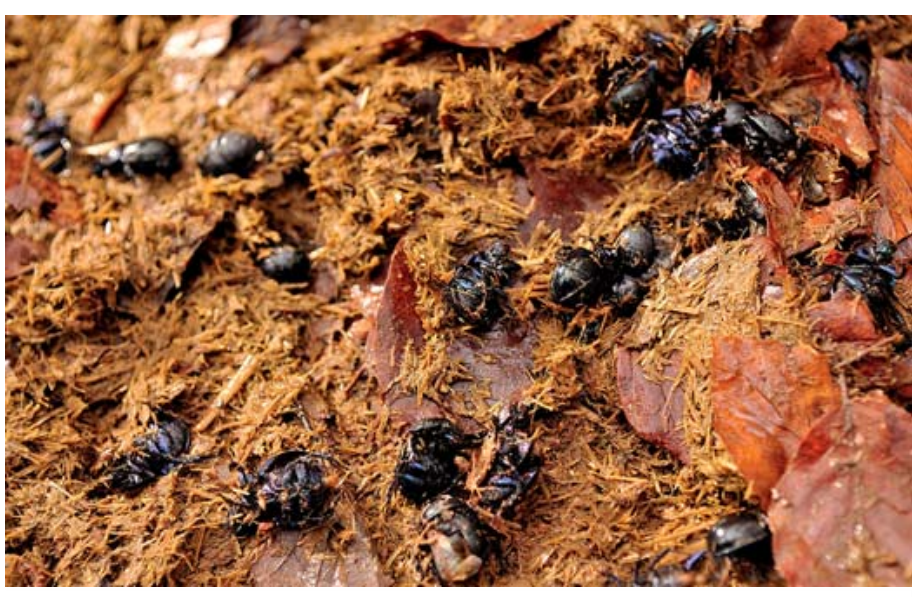

- Abb. 1 Ivermectin wird zu über $50 \%$ unverändert über den Kot ausgeschieden und wirkt dort noch bis zu 3 Wochen tödlich auf Nematoden und Arthropoden [20]. Für Mistkäfer werden in der Natur abgesetzte Pferdeäpfel nach Ivermectin-Therapie zur tödlichen Falle. @ Ferdinand Worm

\section{Resistenzentwicklung}

Vor allem bei den als Vektoren besonders wichtigen Zecken wird weltweit eine Zunahme von Resistenzen gegen Ektoparasitika festgestellt [10]. Aber auch Insekten können gegen die genannten Stoffe Resistenzen entwickeln. Selbst Mehrfach-, Gruppen- und Kreuzresistenzen können auftreten. Begünstigt werden Resistenzentwicklungen durch die Verwendung von zur Langzeitwirkung konzipierten Produkten wie Halsbänder (Hund, Katze) und Ohrclips (Rind) mit puder- oder gasförmigen Formulierungen und durch hohen, langanhaltenden Selektionsdruck durch häufige Anwendung [20]. Resistenzen bei Ektoparasiten gefährden insbesondere Menschen und Tiere in solchen Regionen der Erde, in denen diese nicht nur lästig sind, sondern auch als Krankheitsüberträger große Bedeutung haben. Das sollte Anlass zu verantwortungsbewusstem Umgang mit diesen Stoffen geben.

Merke

Umgang mit Zeckenbefall, wenn höchstwahrscheinlich keine lebensbedrohlichen Erreger übertragen werden:

- Kein prophylaktisches Anwenden von Akariziden

- Bei starker Neigung zu Zeckenbefall nach einem evtl. zugrunde liegenden Problem suchen (v.a. Kontrolle der Darmgesundheit, Leber- und Nierenfunktion).

- Ist eine Bekämpfung nötig, sollten zuerst pflanzliche Präparate versucht werden.

\section{Parasitenprophylaxe}

Sowohl die potenzielle Toxizität von Antiparasitika für den Warmblüter als auch die Ökotoxizität und die Resistenzproblematik müssen in der Tierärzteschaft zu kritischem Hinterfragen gängiger Vorgehensweisen wie Anwendung im vierwöchigen Abstand ohne Nachweis der Notwendigkeit und Behauptungen wie „unschädlich für 
Mensch und Tier“ führen. Dies gilt gleichermaßen für Ekto- wie Endoparasitika.

Es sollte Aufgabe der Tierärzte sein, der z. T. aus marktstrategischen Gründen geschürten Angst vor Parasiten und der ungerechtfertigten Verharmlosung von Antiparasitika durch sachliche Information des Tierhalters entgegenzutreten. Zu einer guten Beratung des Tierbesitzers gehört diesbezüglich neben der Aufklärung über die tatsächlichen Risiken von Parasiten und Antiparasitika auch das Informieren über alternative Therapeutika, die zwar i.d. R. weniger anwenderfreundlich und effizient sind, dafür jedoch auch weniger toxisch für Anwender, Tier und Umwelt. Besonders wichtig ist zudem die Anleitung zu sinnvollem Management in der Parasitenbekämpfung (s.u.).

\section{Parasitenabwehr phytotherapeutisch}

Da Pflanzen ebenso wie Tiere von Parasiten bedroht werden, findet man auch bei ihnen antiparasitär wirksame Inhaltsstoffe, die verhindern, dass der Parasit überhandnimmt. Dabei handelt es sich i.d.R. um einen reichhaltigen „Cocktail“ von Wirkstoffen, der im Zuge der Evolution immer wieder in der Auseinandersetzung mit dem Fraßfeind optimiert wurde und wird. Dass Parasiten vollständig resistent werden, wie es bei auf wenige Targets optimierten synthetischen Antiparasitika vorkommt, ist bei diesen sich ständig ändernden Vielstoffgemischen höchst unwahrscheinlich. Weltweit nutzen Wildtiere die Pflanzen ihres angestammten Biotops, um Endo- und Ektoparasiten unter Kontrolle zu halten. Beobachtungen zur Selbstmedikation von Tieren mittels Pflanzen gegen Parasiten wurden in den letzten Jahren zahlreich dokumentiert $[11,16,17]$. Dies geschah nicht zuletzt, um Wirkstoffen auf die Spur zu kommen, die aus der Misere der Resistenzentwicklung bei den synthetischen Antiparasitika helfen könnten. Häufig sind ebendiese Pflanzen auch in der Volksmedizin als Antiparasitika bekannt. In Ländern mit niedrigen Standards bei öffentlicher Hygiene und medizinischer Versorgung werden Pflanzen auch heute noch gegen Parasiten eingesetzt. Unter diesen Pflanzen sind diverse Arzneipflanzen mit z.T. Jahrhunderte alter Anwendungstradition, die mit dem Aufkommen synthetischer Produkte bedeutungslos wurden. Aufgrund der sich ausbreitenden Resistenzen gegen Synthetika und der wachsenden Erkenntnis, dass die Zielsetzung, Parasiten auszurotten, technisch nicht möglich und medizinisch nicht sinnvoll ist (s. Kasten S.101), werden diese Arzneipflanzen heute wieder interessant. Man denke nur an die „Karriere“ des Einjährigen Beifuß, Artemisia annua, der heute als Malariamittel hohe Wertschätzung genießt und auch bei anderen Blutparasitosen beachtenswertes Potenzial zeigt, wie z. B. bei der Leishmaniose [22].

An dieser Stelle sollen exemplarisch die Dalmatinische Insektenblume und der Neembaum vorgestellt werden.
Dalmatinische Insektenblume, Tanacetum cinerariifolium (Familie der Asteraceae)

Schon seit der römischen Antike verwendet man die Blüten der Dalmatinischen Insektenblume, das sogenannte „Persische Insektenpulver“ gegen Läuse und Flöhe. Seit Ende des 19. Jh. wurden Extrakte der Dalmatinischen Insektenblume, das Pyrethrum, verstärkt in verschiedenen Bereichen der Landwirtschaft als natürliches Kontaktinsektizid eingesetzt. Die Pflanze stammt ursprünglich aus der Adriaküstenregion. Kultiviert wird sie heute in Afrika (v.a. Kenia), Asien und Südamerika. Der Extrakt enthält als Hauptwirkstoffe insektizid wirksame Pyrethrine. Diese wirken am Wirtstier nur kurzfristig parasitenabtötend und ansonsten v.a. als Repellent. Sie werden vom Wirt in geringen Mengen über die Haut resorbiert und können nach Glucuronidierung renal ausgeschieden werden. Die Dalmatinische Insektenblume und ihre Zubereitungen sollten bei Katzen möglichst nicht angewendet werden (Glucuronidierungsschwäche). Pyrethrum wird zumeist als Biozid zur Anwendung in der Umgebung zugelassen $[15,20]$.

Die jüngere Geschichte des Pyrethrums - nämlich seine chemische Veränderung zu Pyrethroiden - ist ein Beispiel für die Unabsehbarkeit der Folgen menschlichen Eingreifens und kurzsichtiger Risikobewertung.

\section{VOM PYRETHRUM ZUM PYRETHROID}

Die Pyrethrine zersetzen sich im Tageslicht rasch, wodurch sie ihre Toxizität verlieren. Zur Verbesserung der Haltbarkeit und damit zur Wirkungsverlängerung wurden sie mit dem Synergisten Piperonylbutoxid kombiniert. Dieser ist ein Cytochrom P450-Inhibitor und verhindert die Metabolisierung der Pyrethrine - sowohl im Parasiten als auch im Wirt. Chemisch veränderte Pyrethrine, die Pyrethroide wie Permethrin, Cypermethrin und Deltamethrin sind wirksamer in der Parasitenbekämpfung, haben aber auch eine höhere Ökotoxizität durch lange Persistenz und können beim Wirt durch Kumulation nach Resorption zu einer chronischen Pyrethroidbelastung führen. Diese äußert sich beim Menschen in sensomotorischer Polyneuropathie, Konzentrationsund Gedächtnisstörungen, mangelndem Durchhaltevermögen, Antriebslosigkeit, Verlust der Lebensfreude und sozialem Rückzug (Neurotoxisches Syndrom) [21]. Beim Menschen wird zudem ein Zusammenhang mit dem Auftreten von Morbus Parkinson vermutet. In Frankreich wird Morbus Parkinson bei Landwirten, die nachweislich mehr als 10 Jahre mit Pestiziden Kontakt hatten (unter denen Pyrethroide einen hohen Marktanteil haben!), als Berufskrankheit anerkannt. Pyrethroide sind in der Tiermedizin i.d. R. verschreibungspflichtige Arzneimittel. 


\section{Neembaum, Azadirachta indica; syn. Melia azadirachta}

Der Neembaum stammt aus den tropischen Regionen Indiens, Sri Lankas und Indonesiens, kommt in Australien und Westafrika vor. In der indischen Landwirtschaft bekämpft man seit langem mit wässrigen Zubereitungen aus dem Samenschrot des Neembaums Insekten, Nematoden, Milben und Pilzinfektionen. Das Öl der Fruchtkerne, das sog. Neemöl, syn. Margosa-Öl, findet heute breite Anwendung im Pflanzenschutz des Biologischen Landbaus. Neemöl ist als repellentes Biozid registriert. Es wird bei allen Tierarten angewendet und hat sich z. B. zur Bekämpfung der Schafslausfliegen („Wollläuse“) durch Sprüh- oder Badebehandlung bewährt. Insektizider Inhaltsstoff ist das Azadirachtin. Weitere Inhaltsstoffe sind diverse repellent wirkende Triglyceride wie Ölsäure, Stearinsäure, Linolsäure und Palmitinsäure. Neemöl wirkt antiparasitär, repellent (Mücken), anthelmintisch, desinfizierend und heilungsfördernd. Das Azadirachtin im Neemöl ähnelt in seiner Wirkung dem für die Häutung von Insekten zuständigen Hormon Ecdyson. Es hemmt die Larvenentwicklung der Insekten (Fraßgift).

Neemöl kann z. B. äußerlich als Waschung angewendet werden. Hierzu werden $25 \mathrm{ml}$ Neemöl in $400 \mathrm{ml}$ Shampoo eingearbeitet. Neemöl ist als Biozid erhältlich, auch in Kombination mit ätherischen Ölen.

Gegenanzeigen für Neemöl sind Gravidität und Laktation. Neemprodukte sollten bei der Katze vorsichtig eingesetzt werden. Es wurden wiederholt Vergiftungen durch Neemöl mit Symptomen wie Hypersalivation, Ataxie, Zittern und Krämpfen beschrieben [20].

\section{Ätherische Öle}

Es gibt eine Vielzahl traditionell als Repellentien sowie als Insektizid und Akarizid eingesetzter ätherischer Öle. Zumeist schützen diese ätherischen Öle nur ca. 1 Stunde. Gute repellente Wirkung geht vom Geraniol, einem Monoterpen, aus. Geraniol wirkt über mehrere Stunden und ist in vielen ätherischen Ölen als wesentliche Komponente enthalten. Vorsicht: Ätherische Öle werden durch die Haut resorbiert! Sie sind für Katzen nicht geeignet.

Das bei der Gewinnung des ätherischen Öls vom Zitroneneukalyptus (Corymbia citriodora, syn. Eucalyptus citriodora) aus dem Destillationsrückstand gewonnene pMenthane-3,8-diol (PMD; cis- und trans-Isomere des Citronellals, Handelsname Citriodiol) wirkt mehrere Stunden repellierend (nicht flüchtig) [32].

Merke

Repellent, insektizid und akarizid wirksame Ätherischöl-Drogen (Auswahl) [15]

Myrtaceae:

- Gewürznelken, Syzygium aromaticum

- Eukalyptus, Eucalyptus globulus

- Teebaum, Melaleuca alternifolia

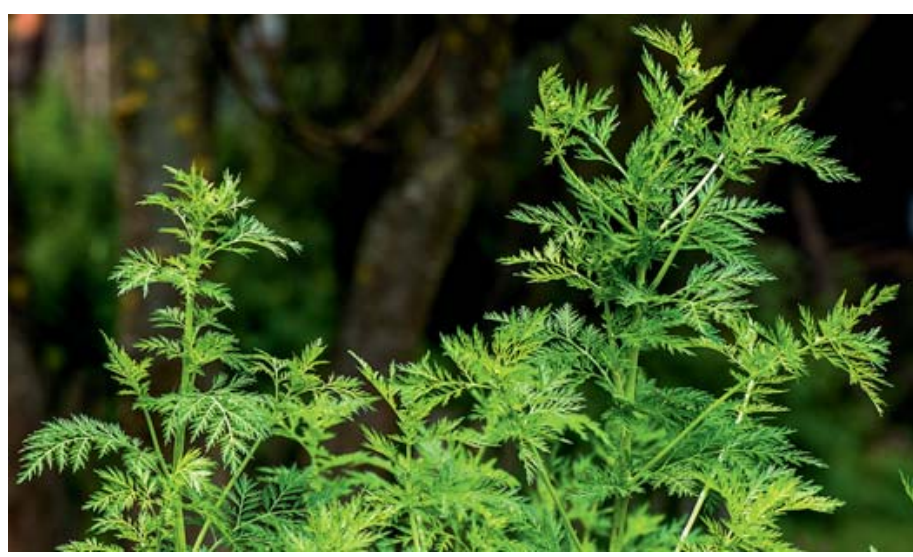

- Abb. 2 Klimatische Veränderungen, globaler Handel mit Tieren und Tourismus begünstigen die Ausbreitung von Blutparasiten wie Leishmanien. Artemisia annua, Einjähriger Beifuß, in China seit Jahrtausenden probates Malaria-Therapeutikum, kann hier erfolgreich eingesetzt werden [22]. (C) Ferdinand Worm

Pinaceae:

- Zeder, Cedrus atlantica

Geraniaceae:

- Rosen-Pelargonie, Pelargonium graveolens

Lamiaceae:

- Lavendel, Lavandula angustifolia

- Pfefferminze, Mentha x piperita

- Thymian, Thymus vulgaris

Poaceae:

- Lemongrass (syn. Zitronengras, Indische Melisse), Cymbopogon citratus

- Palmarosagras, Cymbopogon martinii

- Citronellagras, Cymbopogon nardus

FORSCHUNGSERGEBNISSE ZUR REPELLENTEN UND INSEKTIZIDEN BZW. AKARIZIDEN WIRKUNG VON ÄTHERISCHEN ÖLEN (AUSWAHL)

Anopheles-Mücken

In Studien konnte belegt werden, dass die ätherischen Öle aller drei Cymbopogon-Arten einen weitreichenden Schutz (> 90\%) vor Anopheles-Mücken bieten. Dies gilt insbesondere für Anopheles culicifacies, dem wichtigsten Vektor der Malaria in Indien. Die repellente Wirkung der ätherischen Öle der Cymbopogon-Arten war mit der chemischer Repellentien vergleichbar [5].

Rote Vogelmilbe

In Studien konnte gezeigt werden, dass das ätherische Öl des Palmarosagrases als Akarizid eingesetzt werden kann. Es erwies sich in einer Konzentration von $0,14 \mathrm{mg} / \mathrm{cm} 3$ als zu $84 \%$ tödlich für die Rote Vogelmilbe (Dermanyssus gallinae). Die repellierende Wirkung setzte bereits 45 Minuten nach der Anwendung ein und dauerte bis zu 5 Tage. Auch das ätherische Öl des Thymians kann als Akarizid gegen die Rote Vogelmilbe eingesetzt werden. Es erwies sich ebenfalls in einer Konzentration von $0,14 \mathrm{mg} / \mathrm{cm} 3$ als zu $84 \%$ tödlich für die Milbe. Die repellierende Wirkung dauerte bis zu 13 Tage [13]. 


\section{„PARASITEN-OVERKILL“, ALLERGIE UND ATOPIE}

\section{Evolutionäres Wettrüsten}

Parasit und Wirt beeinflussen sich gegenseitig in ihrer Entwicklung. Der Wirt reagiert auf den Parasiten mithilfe seines Immunsystems. Der Parasit muss den immunologischen Reaktionen seines Wirtes ausweichen, um sich fortpflanzen zu können. Diese Interaktion zwischen Wirt und Parasit nennt sich Ko-Evolution und gleicht einem Wettrüsten. Parasiten steigern demnach die Immunleistung ihres Wirtes.

\section{Allergie und Atopie}

Weltweit nimmt die Zahl der Allergiker zu. Dieser Trend zeigt sich vor allem in Industrieländern mit hohem Hygienestandard. Viele Jungtiere (v. a. Hunde und Katzen) und viele Kinder dieser Regionen kommen nur noch selten mit Parasiten und Infektionserregern in Berührung. Folglich wird ihr Immunsystem diesbezüglich nur noch selten gefordert.

Die Aufgabe des Immunsystems besteht darin, Fremdstoffe und Krankheitserreger zu erkennen und zu eliminieren. Das funktioniert auch bei Allergikern. Doch es gibt einen gravierenden Unterschied: Statt eine Toleranz gegenüber unschädlichen Substanzen zu entwickeln, fahren Allergiker ein riesiges Waffenarsenal auf, um Fremdstoffe (Allergene) aus dem Feld zu schlagen. Es wird eine Immunantwort induziert, die den Körper schädigt. Nach jedem neuen Kontakt mit dem bereits bekannten Auslöser treten die überzogenen Reaktionen wieder auf. Bei den meisten Allergien spielen hier IgE-Antikörper, die normalerweise der Abwehr von Endoparasiten dienen, die Hauptrolle. Gesunde haben nur sehr niedrige IgE-Spiegel. Allergiker hingegen produzieren große Mengen IgE.

\section{Entstehung einer Allergie}

Eine Allergie entsteht in zwei Phasen. In der ersten Phase - während der sogenannten Sensibilisierung - kommt die Immunabwehr zum ersten Mal mit potenziell allergenen Stoffen, z. B. Pollen, in Berührung. Schon Spuren davon genügen, damit als Abwehrreaktion gegen diese eigentlich harmlosen Fremdstoffe lgE in großer Menge gebildet werden. In dieser ersten Phase hat der Allergiker in der Regel noch keine außergewöhnlichen Beschwerden. In der zweiten Phase führt jeder weitere Kontakt mit demselben Stoff (Allergen) zu einer allergischen Reaktion: Mastzellen schütten entzündungsfördernde Substanzen wie Histamin aus. Schlagartig weiten sich die Gefäße. Die Schleimhäute schwellen an, die Schleimbildung wird angeregt und vieles mehr.

Auch bei der Atopie, einer erblich prädisponierten, durch Umweltfaktoren beeinflussbaren endogenen Hyperreagibilität des Immunsystems, treten vermehrt lgE auf.

\section{Allergien und Umweltfaktoren}

Für den Menschen konnte in Studien gezeigt werden, dass bei einer gesunden, möglichst naturbelassenen Ernährung und Auseinandersetzung mit Bakterien und Endoparasiten in den ersten Lebensjahren Allergien seltener auftreten. Das Aufwachsen unter unterdurchschnittlichen hygienischen Bedingungen, mit mehreren Geschwistern und der Kontakt mit Natur und anderen Tieren ist mit dem Auftreten von Allergien negativ korreliert.

\section{Die Bedeutung der Mikroflora}

Während und kurz nach der Geburt gelangen Bakterien aus der Keimflora der Mutter und der Umwelt in das Neugeborene und prägen die Zellen seines Immunsystems. Wenn das Zusammenspiel von Immunsystem und Mikroorganismen in dieser sensiblen Phase gestört wird (z. B. durch Sectio caesarea, Desinfektion oder Antibiose), hat dies lebenslange Folgen, die bisher nur unzureichend erforscht sind. Auch im späteren Leben behält die Mikroflora wesentlichen Einfluss auf das Immunsystem. Sie dient dem permanenten Training immunologischer Funktionen.

\section{Die Bedeutung von Magen-Darmparasiten}

Magen-Darmparasiten aktivieren Typ2-T-Helferzellen (TH2- Lymphozyten, Th2), die für die humorale Immunantwort verantwortlich sind. Frühe Parasitosen dienen dem Organismus offenbar als Übung für eine regelrechte Th2-Antwort. Parasiten haben vermutlich aufgrund der Tatsache, dass sie dem Immunsystem ein adäquates Substrat zum Training der Th2-Antwort bieten, eine allergievermeidende Wirkung. So kommt es nicht oder zumindest seltener zu inadäquaten Reaktionen auf harmlose Pollen oder Nahrungsbestandteile. Durch die Th2-Antwort wird viel IL-4 und IL-10 gebildet, die beide anti-inflammatorisch wirken. Diese stehen beim gesunden Immunsystem in Balance mit den durch die Th1-Reaktion (zelluläre Immunantwort) induzierten IL-12 und Interferon-gamma, die maßgeblich an der Aufrechterhaltung der zellulären Abwehr und Entzündungsprozessen beteiligt sind. Der britische Immunologe Graham Rook plädiert deswegen dafür, Parasiten nicht als Bösewichte anzusehen, sondern als „gute alte Freunde“ [27].

\section{Fazit}

Den besten Schutz vor der Entstehung von Allergien bieten nach derzeitigem Kenntnisstand die Abkehr von übertriebener Hygiene und der bewusst provozierte Kontakt mit der belebten Umwelt mit ihren Mikroorganismen bereits beim Neugeborenen. Unter diesen Aspekten ist das derzeit übliche Vorgehen gegen Parasiten zu überdenken. 


\section{Diatomeenerde}

Eine erfahrungsgemäß gut wirksame und nicht toxische Alternative zu Phytotherapeutika, geeignet für Katzen und Geflügel (z. B. im Staubbad der Hühner) ist feinst vermahlene Diatomeenerde. Diatomeenerde, syn. Kieselgur, besteht aus den Schalen fossiler Kieselalgen. Das sehr poröse Material besteht hauptsächlich aus Siliciumdioxid (SiO2). Seine insektizide/akarizide Wirkung kommt vermutlich mechanisch durch Austrocknung der Tracheen und Immobilisierung der Beingelenke bei den Parasiten zustande. Der Vorteil der Diatomeenerde: es kommt nicht zur dermalen Resorption durch den Wirt.

\section{Die Bekämpfung von Endoparasiten mit Sinn und Verstand}

Keine Frage: hochgradiger Befall mit Endoparasiten kann zu schweren Erkrankungen und sogar zum Tod führen, wenn er nicht therapiert wird. Umso bedrohlicher ist die weltweit zunehmende Resistenzentwicklung bei MagenDarmwürmern gegen diverse Anthelminthika (v. a. Benzimidazole, Avermectine). Sogar Multiresistenzen werden beobachtet. Diese betreffen alle domestizierten Tierarten und letztlich auch den Menschen. Verursacht werden sie, ähnlich wie bei den Antibiotika, insbesondere durch Anwendungsfehler.

\footnotetext{
Merke

Anwendungsfehler als Ursache für Resistenzentwicklungen bei Parasiten:

- häufiges (regelmäßiges) Entwurmen ohne nachgewiesene Notwendigkeit

- Entwurmungen ohne spätere Erfolgskontrolle

- Entwurmung mit dem Ziel der Wurmfreiheit
}

Mit Ausnahme großer Nutztierbestände, in denen nur Stichproben und die Beprobung verdächtiger Tiere möglich sind, sollte der Nachweis der Notwendigkeit einer Entwurmung bei jedem einzelnen Tier erbracht werden. Wird der Erfolg einer Entwurmung nicht systematisch kontrolliert, leistet dies der Resistenzentwicklung Vorschub, da ein Wirkungsverlust des Anthelminthikums zu spät wahrgenommen wird. Wurmfreiheit kann nur unter laborähnlichen Bedingungen erreicht werden, wie sie z. B. in geschlossenen Schweinebeständen oder bei ausschließlicher Wohnungshaltung von Heimtieren annähernd gegeben sind. Wurmfreiheit sollte aus medizinischen Erwägungen jedoch gar nicht angestrebt werden, da Parasiten für ein normal reagierendes Immunsystem eminent wichtig sind (TH1/TH2-Balance; s. Kasten S. 100).

Es ist hinreichend belegt, dass ein Immunsystem, dem als „Sparringspartner“ die Parasiten fehlen, zur Hyperreaktivität neigt. Dies erklärt, warum ausgerechnet Parasitenzubereitungen, z. B. aus Trichuris suis, beim Menschen be- reits seit Jahren erfolgreich gegen Autoimmunerkrankungen wie IBD, Morbus Crohn, Colitis ulcerosa, Asthma u. a. eingesetzt werden.

\section{Selektive Entwurmung}

Für Pferde propagieren einige Parasitologen seit vielen Jahren die „Selektive Entwurmung“. Diese basiert auf einem fortlaufenden Monitoring durch diagnostische Kotproben vor geplanten und nach durchgeführten Entwurmungen, bei denen die Wurmei- bzw. Wurmlarvenausscheidung des einzelnen Tieres oder des ganzen Bestandes überprüft, dokumentiert und bewertet wird. Solange ein Pferd nicht mehr als 200 Eier (insbes. kleine Strongyliden) pro g Kot ausscheidet, wird dieses Pferd nicht entwurmt. Ein solch geringgradiger Wurmbefall verursacht keine klinischen Symptome und wirkt positiv auf das Immunsystem. Entschließt man sich wegen höherer Ei-Ausscheidung zu entwurmen, wird nach 2 Wochen erneut eine Kotuntersuchung zur Wirksamkeitskontrolle durchgeführt. Nach weiteren 4 Wochen sollte nochmals der Kot kontrolliert werden, um sicherzustellen, dass nicht wieder eine massenhafte Vermehrung der Parasiten stattgefunden hat [14]. Tiere, bei denen dies der Fall ist, brauchen neben einem Anthelminthikum dringend langfristige Unterstützung beim Aufbau eines widerstandsfähigen Darmmilieus. Hier bietet die Phytotherapie gute Ansätze ( $\vee$ Tab. 1; S. 103).

Durch dieses Vorgehen, das sich auf andere Tierarten entsprechend angepasst übertragen lässt, kann der Einsatz von Anthelminthika drastisch reduziert werden. Dadurch reduziert sich auch der Selektionsdruck hin zu Anthelminthika-resistenten Parasiten deutlich. Die Tiere werden weniger durch Antiparasitika und Immunstörungen wie Allergien belastet.

\section{ZUSATZINFO}

Die Resistenzlage gegen Antiparasitika verschärft sich im gleichen Ausmaß wie die Resistenzentwicklung bei Bakterien gegen Antibiotika. Das ist zum einen fatal für die Fälle, in denen Parasiten nicht nur lästig, sondern lebensgefährlich sind bzw. in denen sie als Krankheitsüberträger fungieren. Zum anderen kann es die Weidehaltung von Nutztieren unmöglich machen, wie dies u. a. Schafhalter in Australien schon erleben mussten.

\section{Wissensvermittlung durch den Tierarzt}

Leider interessiert Tierbesitzer mehrheitlich nur der Preis von „Wurmkuren“. Mit dem preisgünstigen Verkaufen ist es jedoch für uns Tiermediziner nicht getan. Wir sind verpflichtet, für einen verantwortungsvollen Umgang mit Antiparasitika zu sorgen. Dazu gehört, dass wir dafür sorgen, dass auch der Tierbesitzer sich seiner Verantwor- 
tung bewusst wird. Richtiger Umgang mit Antiparasitika setzt Wissen voraus, das wir vermitteln können:

- Gesunde Tiere entwickeln im Laufe des Lebens eine natürliche Immunität gegen Magen-Darm-Parasiten. Entwurmungen sind dann nur noch in seltenen Fällen indiziert.

- Die Vorstellung, dass mit synthetischen Anthelminthika eine „prophylaktische Entwurmung“ möglich ist, ist falsch. (Dies wird offenbar bereits durch den Begriff „Wurmkur“ suggeriert, der deshalb vermieden werden sollte.)

- Nicht das Tier ist ggf. resistent gegen ein Antiparasitikum, sondern dessen Parasiten. Dies ist zur Vermeidung der Kontamination eines Bestandes oder der
Umgebung durch Tiere mit resistenten Parasiten besonders wichtig zu wissen.

- Eine erfolgreiche Parasitenkontrolle setzt voraus, dass man die Lebenszyklen und die Wirtsspezifität der Parasiten kennt.

- In der Nutztier- und Pferdehaltung entscheidet v.a. das Weidemanagement über den Erfolg der Parasitenbekämpfung.

Gerade in der Nutztierhaltung spielen Endoparasiten eine große Rolle. Hohe Besatzdichten ziehen hohen Infektionsdruck nach sich. Zudem fehlen dem Futter unserer Haustiere zumeist die zur Parasitenminimierung notwendigen bioaktiven Futterpflanzen. Dadurch wird die Vitalität und die Darmgesundheit der Tiere negativ beeinflusst

\section{ZUSATZINFO}

\section{Entwurmung vor 120 Jahren [12]}

In der traditionellen veterinärmedizinischen Literatur stößt man auf eine Vielzahl antiparasitär wirksamer Arzneipflanzen. Allerdings sind viele dieser Pflanzen toxisch und nach heutigen arzneimittel- und futterrechtlichen Bestimmungen nicht mehr anwendbar.

\section{Artemisia cina O.C. Berg}

Als wichtigstes Mittel gegen Spulwürmer bei kleinen Haustieren galten die Blütenknospen von Artemisia cina („Wurm“- o. „Zitwersamen“) aus Mittelasien bzw. das daraus gewonnene Santonin in Kombination mit Rizinusöl (resorptionsmindernd, Abführen der nur gelähmten Würmer). Schon in therapeutischen Dosen konnten neben Koliken epileptiforme Krämpfe, Schlafsucht, Lähmungen etc. auftreten. Besonders empfindlich reagierten Jungtiere. Artemisia cina wird noch heute als Homöopathikum bei Wurmbeschwerden eingesetzt.

\section{Dryopteris filix mas (L.) Schott (syn. Aspidium filix mas)}

Eugen Fröhner schreibt [12]: „Die Wurmfarnwurzel ist das älteste und beste Bandwurmmittel (...). Es muss der Farnwurzel 3-6 Stunden nach der Verabreichung ein Abführmittel nachgeschickt werden, weil die Bandwürmer häufig nur vorübergehend betäubt werden (...). Endlich darf nicht außer Acht gelassen werden, dass das Extractum Filicis (Anm: Etherextrakt des Wurmfarnrhizoms) sehr giftig ist. Auch aus diesem Grunde empfiehlt es sich, (...) immer ein Abführmittel nachzugeben... (...) jedoch Ricinusöl hiebei zu vermeiden.“ Zu den wesentlichen toxischen Wirkungen gehörten: Affektion von Gehirn und Rückenmark mit Apathie, Bewusstlosigkeit, auch Erregung, Zwangsbewegungen, Lähmungen, Nystagmus, Mydriasis, Blindheit, parenchymatöse Nephritis.

\section{Mallotus philippinensis (Kamala)}

Der Kamalabaum, Mallotus philippinensis, kommt in Äthiopien, Südarabien, im tropische Asien, nordöstlichen Australien, Neu-Guinea und den Philippinen vor.

Kamala, die von der Frucht des Kamalabaumes abgeriebenen Haarorgane, diente nachweislich schon 500 v. Chr. zu kultischen Zwecken und als Färbemittel für Seide, wozu es noch heute im Handel ist. 1841 wurde erstmalig über Kamala als wurmtreibendes Mittel berichtet. 1882 wurde Kamala ins deutsche Arzneibuch aufgenommen. Fröhner empfahl Kamala für Kleintiere und Geflügel als „vorzügliches Antitänicum, welches gleichzeitig drastisch wirkt, sodass bei ihrer Anwendung die spätere Verabreichung eines Abführmittels erspart wird“. Auch gegen Spulwürmer hielt er Kamala für wirksam. Der Abgang der Bandwürmer sei nach durchschnittlich 5 Stunden zu erwarten. Kamala wurde in Milch eingerührt. Fröhners Nachfolger als Autor der Arzneimittellehre für Tierärzte, Richard Reinhardt [26], erwähnt 1950 Kamala auch zur Anwendung gegen Fasciola hepatica beim Schaf. Gegen Dicrocoelium lanceolatum sei Kamala nach seinem Wissen nicht wirksam. Er warnt zudem vor der Anwendung von Kamala gegen die Faszielose beim Rind; hier sei Kamala ein unsicheres, ungeeignetes und gefährliches Mittel. F. R. Ungemach bezeichnet Kamala 1994 als obsolet [19]. Die für Kamala beanspruchten Indikationsgebiete von Darmnematoden und Darmcestoden bei allen Tierarten hält er weder in ihrer Wirksamkeit für bewiesen, noch sei die Verträglichkeit ausreichend belegt. Kamala enthält u. a. Phloroglucinderivate, Glykoside, Gerbsäure und organische Säuren. Sie wirkt anthelminthisch und peristaltikanregend [15].

Fröhner empfahl für große Hunde 5-15 g, für kleine Hunde $2-5 \mathrm{~g}$, für Katzen und großes Geflügel 1-2 g, für kleineres Geflügel (Tauben, Papageien) 0,5-1 g [12]. Reinhardt gab als Dosierung für Schafe 7,5 g, 2 x im Abstand von 12-24 Stunden und für Rinder $15 \mathrm{~g}$, wöchentlich mehrfach zu wiederholen bei Spulwurmbefall an [26]. Kamala ist zurzeit für mehrere Tierarten als Ergänzungsfuttermittel in Kombination mit anderen Pflanzenstoffen im Handel. 
- Tab. 1 Arznei- und Futterpflanzen mit positivem Einfluss auf die Darmgesundheit und mit antiparasitärer Wirkung (Auswahl).

\begin{tabular}{|c|c|c|c|}
\hline $\begin{array}{l}\text { Pflanzenname dt./lat. } \\
\text { verwendeter Pflanzenteil }\end{array}$ & wesentliche Inhaltsstoffe & Wirkungen (Auswahl) & Bemerkungen \\
\hline $\begin{array}{l}\text { Curcuma (syn. Gelbwurz) } \\
\text { Curcuma longa } \\
\text { Javanische Gelbwurz } \\
\text { Curcuma zanthorrhiza } \\
\text { Familie: Zingiberaceae } \\
\text { Wurzelstock }\end{array}$ & $\begin{array}{l}\text { ätherisches Öl } \\
\text { Curcuminoide } \\
\text { Stärke }\end{array}$ & $\begin{array}{l}\text { verdauungsfördernd } \\
\text { choleretisch } \\
\text { hepatoprotektiv } \\
\text { immunmodulierend } \\
\text { (antiparasitär) }\end{array}$ & $\begin{array}{l}\text { In der Kombination mit Pfeffer wird die biologi- } \\
\text { sche Halbwertszeit der Curcuminoide um ein } \\
\text { Vielfaches verlängert, weil das Piperin im Pfeffer } \\
\text { die Glucuronidierung hemmt, die zur Ausschei- } \\
\text { dung der Curcuminoide nötig ist [29]. }\end{array}$ \\
\hline $\begin{array}{l}\text { Ingwer } \\
\text { Zingiber officinale } \\
\text { Familie: Zingiberaceae } \\
\text { Wurzelstock }\end{array}$ & $\begin{array}{l}\text { ätherisches Öl } \\
\text { Arylalkanone (Scharfstoffe) } \\
\text { Curcuminoide } \\
\text { Stärke }\end{array}$ & $\begin{array}{l}\text { verdauungsfördernd } \\
\text { spasmolytisch } \\
\text { Steigerung MD- Tonus u. Peris- } \\
\text { taltik } \\
\text { antiparasitär }\end{array}$ & $\begin{array}{l}\text { Ingwer hat sich in Studien als wirksam gegen } \\
\text { Askaridia galli und Giardien erwiesen }[6,8] \text {. Die } \\
\text { Wirkung ist konzentrations- und zeitabhängig. }\end{array}$ \\
\hline $\begin{array}{l}\text { Esparsette } \\
\text { Onobrychis viciifolia } \\
\text { Familie: Fabaceae } \\
\text { ganze Pflanze }\end{array}$ & $\begin{array}{l}\text { Gerbstoffe, v. a. Proanthocyanidi- } \\
\text { ne, kondensierte Gerbstoffe } \\
\text { Aminosäuren } \\
\text { Flavonoide, v. a. Rutin } \\
\text { Arbutin }\end{array}$ & antiparasitär & $\begin{array}{l}\text { Bei Ziegen verringert sich ca. } 3 \text { Wochen nach } \\
\text { Fütterungsbeginn mit Esparsette (30-40\% als } \\
\text { dauerhafter Ersatz der sonstigen Futterration) die } \\
\text { Eiausscheidung der MD-Würmer um bis zu } 50 \% \text {. } \\
\text { Die Würmer werden nicht getötet, nur die Eiaus- } \\
\text { scheidung wird beeinträchtigt. Erst bei höheren } \\
\text { Mengen sterben die Würmer ab (Cave: antinutri- } \\
\text { tive Effekte der Gerbstoffe). Im Gegensatz zu an- } \\
\text { deren Leguminosen verursacht Esparsette auf- } \\
\text { grund des Gerbstoffgehaltes keine schaumige } \\
\text { Gärung }[29,30] \text {. }\end{array}$ \\
\hline $\begin{array}{l}\text { Karotte (syn. Möhre) } \\
\text { Daucus carotus subsp. sativus } \\
\text { Familie: Apiaceae } \\
\text { frische Karotte oder ihr Saft }\end{array}$ & $\begin{array}{l}\alpha-, \beta \text { - und } \gamma \text {-Carotin Pektine } \\
\text { ätherisches Öl } \\
\text { Phytosterole } \\
\text { Vitamine B1, B2 und C }\end{array}$ & $\begin{array}{l}\text { antimikrobiell } \\
\text { anthelminthisch } \\
\text { antidiarrhoisch } \\
\text { fruchtbarkeitssteigernd }\end{array}$ & $\begin{array}{l}\text { Dosierung Karotten/Tag unterstützend bei } \\
\text { Wurmbefall: ca. } 1 \text { kg rohe Karotten } / 500 \text { kg KGW, } \\
250 \mathrm{~g} \text { rohe Karotten/100 kg KGW, } 50 \text { g rohe Ka- } \\
\text { rotten/10 kg KGW [3] }\end{array}$ \\
\hline $\begin{array}{l}\text { Knoblauch } \\
\text { Allium sativum L. } \\
\text { Familie: Amaryllidaceae } \\
\text { Frischer Knoblauch } \\
\text { Knoblauchsaft } \\
\text { Knoblauchtinktur }\end{array}$ & $\begin{array}{l}\text { Alliin als Hauptkomponente } \\
\text { (0,5-2,5\% im Pulver bzw. 0,3- } \\
1,3 \% \text { frisch) } \\
\text { Proteine (u. a. Alliinase) } \\
\text { Steroid- und Tripterpensaponine } \\
\text { Flavonoide } \\
\text { Polysaccharide (Inulin-ähnliche } \\
\text { Fruktane) }\end{array}$ & $\begin{array}{l}\text { verdauungsfördernd } \\
\text { tonisierend } \\
\text { antimikrobiell (u. a. Helico- } \\
\text { bacter pylori, Staph. aureus, } \\
\text { E. coli, Listeria monocytogenes, } \\
\text { Staph. epidermidis, Salmonella } \\
\text { thyphi, Hefen, Cryptoccoccus } \\
\text { neoformans) } \\
\text { immunmodulierend } \\
\text { antiparasitär }\end{array}$ & $\begin{array}{l}\text { Knoblauch ist allein und in Kombination mit an- } \\
\text { deren Arzneipflanzen (u. a. Oregano) in der Lage, } \\
\text { Parasiten wirksam zu kontrollieren. Dies wurde } \\
\text { u. a. für Cestoden, Trematoden (Oxyuren), Ne- } \\
\text { matoden (Haemonchus contortus Schaf), Stron- } \\
\text { gyliden (Pferd), Kokzidien (Kaninchen) und Tri- } \\
\text { chomonaden (Tauben) belegt [1,8,18,23-25, } \\
\text { 28]. }\end{array}$ \\
\hline $\begin{array}{l}\text { Meerrettich (syn. Kren) } \\
\text { Armoracia rusticana (syn. Coch- } \\
\text { learia armoracia) } \\
\text { Familie: Brassicaceae } \\
\text { Meerrettichwurzel }\end{array}$ & $\begin{array}{l}\text { Glucosinolate } \\
\text { Ascorbinsäure } \\
\text { Vitamin } B_{1} \\
\text { Flavonoide }\end{array}$ & $\begin{array}{l}\text { antimikrobiell } \\
\text { hyperämisierend } \\
\text { verdauungsfördernd }\end{array}$ & $\begin{array}{l}\text { Die wurmtreibende Wirkung lässt sich bisher } \\
\text { nicht belegen. Meerrettich trägt jedoch zur Ver- } \\
\text { besserung der Darmgesundheit und damit ver- } \\
\text { mutlich zur Stärkung der natürlichen Resistenz } \\
\text { gegen Wurmerkrankungen bei. } \\
\text { Gegenanzeigen: Magen- und Darmgeschwüre, } \\
\text { Nierenerkrankungen; für Kleintiere, insbes. } \\
\text { Katzen nicht geeignet }\end{array}$ \\
\hline $\begin{array}{l}\text { Schwarzkümmel } \\
\text { (syn. schwarzer Koriander) } \\
\text { Nigella sativa } \\
\text { Familie: Ranunculaceae } \\
\text { Schwarzkümmelsamen } \\
\text { Schwarzkümmelöl }\end{array}$ & $\begin{array}{l}\text { fettes Öl mit > 50\% Glyceriden der } \\
\text { Linolsäure, ca. } 20 \% \text { Ölsäure } \\
\text { ätherisches Öl, v. a. Thymochinon, } \\
\text { p-Cymen, } \alpha \text { - und } \beta \text {-Pinen, } \\
\text { Flavonoltriglykoside } \\
\text { Alkaloide, v. a. Nigellin, Nigellidin, } \\
\text { Proteine } \\
\text { Limonen }\end{array}$ & $\begin{array}{l}\text { antiphlogistisch } \\
\text { antibakteriell } \\
\text { antioxidativ/zellprotektiv } \\
\text { immunmodulierend } \\
\text { antiparasitär }\end{array}$ & $\begin{array}{l}\text { In Indien wurde Schwarzkümmel bereits in den } \\
\text { 1970er Jahren in Tierversuchen mit Ziegen und } \\
\text { Schafen erfolgreich gegen Cestoden eingesetzt } \\
\text { [2]. Bei Kindern wurde die Wirkung von Schwarz- } \\
\text { kümmel bei Cestoden als vergleichbar mit Niclo- } \\
\text { samid gefunden [4]. }\end{array}$ \\
\hline $\begin{array}{l}\text { Wermut (syn. Absinth) } \\
\text { Artemisia absinthium } \\
\text { Familie: Asteraceae } \\
\text { Wermutkraut }\end{array}$ & $\begin{array}{l}\text { ätherisches Öl, u. a. mit } \beta \text {-Thujon } \\
\text { und Sesquiterpen-Bitterstoffen } \\
\text { Phenylpropanderivate } \\
\text { Phenolcarbonsäuren } \\
\text { Flavonoide }\end{array}$ & $\begin{array}{l}\text { appetitanregend } \\
\text { verdauungsfördernd } \\
\text { choleretisch }\end{array}$ & $\begin{array}{l}\text { Wurde traditionell besonders beim WDK gegen } \\
\text { Endoparasiten (Oxyuren) und Ektoparasiten } \\
\text { (Myiasis) eingesetzt. } \\
\text { Wermutkraut ist für Katzen nicht geeignet. Wer- } \\
\text { mut nicht längerfristig einsetzen. }\end{array}$ \\
\hline
\end{tabular}

Angaben zu Dosierungen, Zubereitung und Anwendung siehe: Brendieck-Worm C, Melzig MF (Hrsg.). Phytotherapie in der Tiermedizin. Stuttgart: Thieme Verlag; 2018 [7] 
und es sinkt die Widerstandskraft gegenüber Parasiten, die ihrerseits nicht mehr durch Pflanzenstoffe an einer ungehemmten Vermehrung gehindert werden.

Phytotherapeutika können synthetische Anthelminthika i.d.R. nicht ersetzen (s. auch Kasten Entwurmung vor 120 Jahren). Phytotherapie von Endoparasitosen ist vorrangig als Prophylaxe einer starken Parasitenvermehrung zu sehen: Mit Pflanzeninhaltsstoffen wird Einfluss auf das Darmmilieu genommen und dieses zu Ungunsten der Parasiten verändert. Ein gesunder Darm wiederum bietet beste Voraussetzungen für eine gesunde und widerstandsfähige Haut.

Eine Auswahl von Pflanzen, die zur Stärkung der Darmgesundheit geeignet sind und von denen zudem eine antiparasitäre Wirkung ausgehen kann, zeigt • Tab. 1.

\section{Autorinnen/Autoren}

\section{Cäcilia Brendieck-Worm}

Geb. 1957; 1976-1981 Studium der Veterinärmedizin in Gießen; 1982-1986 Promotion am Institut für Tierzucht und Haustiergenetik der JLU Gießen; 1985-2013 Mitglied einer tierärztlichen Praxis für Groß- und Kleintiere in der Pfalz; Zusatzbezeichnung und Weiterbildungsermächtigung Biologische Tiermedizin; Leitung des Arbeitskreises Phytotherapie der GGTM

\section{Alexandra Nadig}

Selbstständige Tierärztin; Phytotherapeutin und aktives Mitglied der Arbeitsgruppe Phytotherapie der Tierärzte; Mitglied der GGTM; Gründerin der Holispital GmbH, Ganzheitliches

\section{ZUSAMIMENFASSUNG}

Zurzeit werden Parasiten weitgehend schematisch, d. h. ohne Berücksichtigung von Lebenszyklen und Parasitosen begünstigenden Haltungsbedingungen bekämpft. Mögliche Nebenwirkungen für den Wirtsorganismus (v. a. neurologische Spätschäden, Allergieneigung), drohende Resistenzentwicklung bei Parasiten gegen Antiparasitika und die Ökotoxizität der Präparate werden meist nicht thematisiert bzw. treten hinter z. T. irrationalen Ängsten vor Parasiten zurück, die nicht selten aus marktstrategischen Gründen geschürt werden. Tiermediziner können über diese Zusammenhänge aufklären und dem Tierbesitzer zudem Möglichkeiten offerieren, sein Tier durch gesundheitsunterstützende Maßnahmen vor überhandnehmendem Parasitenbefall zu schützen. Dabei steht die Darmgesundheit im Vordergrund, die sich insbesondere durch Phytotherapeutika positiv beeinflussen lässt.
Tiergesundheitszentrum; Gründerin von AnimaPlanta, Heilpflanzenzentrum für Mensch \& Tier

\section{Yvonne Thoonsen}

2000-2007 Studium Veterinärmedizin an der Justus-LiebigUniversität in Gießen; seit 2008 selbstständig in eigener Praxis in Engelskirchen Bickenbach; Mitglied der ATF seit 2008; Mitglied der GGTM seit 2010; Mitglied der GPT seit 2015; ständig aktive Teilnahme in Fort- und Weiterbildungen zu Themen wie z.B. Schmerztherapie, Verhaltenstherapie, Krebstherapie, Osteopathische Akupunktur u.v.m.; seit 2015 in Weiterbildung zur „Biologischen Tiermedizin“, Schwerpunkt Phytotherapie

\section{Korrespondenzadresse}

Dr. med. vet. Cäcilia Brendieck-Worm

Talstraße 59

67700 Niederkirchen

cbw@phyto-fokus.de

\section{Literatur}

Literatur ist in der Online-Version unter www.thieme-connect.de/products einsehbar.

\section{Bibliografie}

DOI https://doi.org/10.1055/a-0634-1981

Zeitschrift für Ganzheitliche Tiermedizin 2018; 32: 96-104

(c) Georg Thieme Verlag KG Stuttgart · New York ISSN 0939-7868

\section{SUMMARY \\ Dealing with Parasites}

Parasites are currently controlled in a largely schematic manner, i.e., without consideration of their respective life-cycles and the husbandry conditions that favor parasitic diseases. The possibility of sideeffects for the host organism (especially neurological sequelae, the disposition to develop allergies), the threat of resistance development in parasites against anti-parasitical medications, and the eco-toxicity of drugs are rarely considered. These aspects are in part neglected due to irrational fears of parasites, which are frequently encouraged out of market-strategy considerations. Veterinarians are able to explain such relationships to animal owners and offer them the possibility to protect their animals from prevalent parasite infestations through health-promoting measures. The main focus is thereby on intestinal health, which can be positively influenced by herbal remedies.

Key words: Parasites, development of resistance, allergies, ecotoxicity 